\title{
Five steps toward improving quality in surgical care
}

\author{
Paul Riss • Thomas Aigmueller
}

Published online: 21 May 2014

(C) The International Urogynecological Association 2014

Quality is an elusive concept and hard to describe. Quality is not, like beauty, in the eye of the beholder, but can be defined and experienced. In surgery, quality means that the right operation is chosen, that everything goes well during and after surgery, and that the desired aim is achieved. While everyone would agree on these points, the actual definition of quality and the selection of benchmark data points are trickier.

Most important of all, however, is the implementation of measures in a hospital system to increase quality. Recently, Dr. Clifford Ko from the American College of Surgeons, in the TeLinde lecture at the 40th Annual Meeting of the Society of Gynecologic Surgeons, presented five observations to increase awareness of what quality actually means. The observations were also meant to assist with "finding the way to achieving surgical quality" - the title of his lecture - and to facilitate the implementation of quality measures in a surgical setting. This is a summary of his presentation.

\section{Observation 1: use good data}

All our considerations must be based on good data. Without good data the outcome is open to question. Good data have three characteristics: they must be accurate, believable, and actionable. In other words they must reflect what actually happened, they must be consistent and make sense, and they

\footnotetext{
P. Riss $(\bowtie)$

Urogynecology and Reconstructive Pelvic Floor Surgery, Division of General Gynecology and Gynecologic Oncology, Department of Obstetrics and Gynecology, Medical University Vienna, Waehringer Guertel 18-20, 1090 Vienna, Austria

e-mail: priuj11@gmail.com

T. Aigmueller

Department of Obstetrics and Gynecology, Medical University Graz, Auenbruggerplatz 14, 8036 Graz, Austria

e-mail: thomas.aigmueller@medunigraz.at
}

must be relevant so that they can be acted upon. One way of making sure that data are valid is to carry out audits. If the difference between the original data and what is determined during an audit is more than $5 \%$, the data are probably not credible.

Another extremely important point is to look at the source of the data. In medicine, very often, administrative data and claims data from insurance companies are used. These data, however, are known to have a poor sensitivity and, for example, generally pick up only $50 \%$ of complications. Administrative data also have a high false-positive rate (58 \% in one study by Lawson et al. [1]). Medical data and data from chart reviews are preferred. Just because data are already there does not mean that they are reliable and that we should use them.

\section{Observation 2: increase evidence-based practice}

We need to better understand the effectiveness and limitations of our clinical research while expanding our use of evidence. Evaluating the evidence and translating it into clinical practice have become the challenge of our times, and there are tools available to make our lives easier. The GRADE method helps to assess evidence [2], and the RAND appropriateness methodology marries best evidence with expert opinion using an independent, non-consensus rating method [3].

The holy grail of the highest-level evidence should not let us forget that it makes eminent sense to include less than level I evidence in our decision-making. It has been shown that in the hierarchy of research designs lower level evidence can be very useful [4, 5]. After all, we are talking about EBPevidence-based practice - which brings together the best research evidence, clinical expertise, and patient values and preferences. 


\section{Observation 3: policy can bring improvements}

Standardization of processes and decision-making can bring real progress toward achieving better quality. The trick is to implement policies in small settings, which increases the chances of success substantially. The point is to agree on a care pathway and to implement it in a team of pelvic floor surgeons in one hospital, and not to wait for national guidelines to become mandatory.

There are two very important caveats. First, improving a process does not necessarily result in better outcomes (and better quality); second, we must distinguish better quality reporting from actual quality. Quality reporting is a means and not an end in itself, and good reporting of quality and good quality are not always synonymous.

\section{Observation 4: equifinality_or "many ways lead to Rome"}

When thinking about quality one has to keep an open mind. One should take as many factors into account as possible while avoiding insisting on one particular process or solution. High-quality evidence may be lacking, clinical expertise may be insufficient, and patient values and preferences may be illdefined - but there may still be another way of achieving the desired goal.

What works in one environment does not necessarily have the same beneficial effect in another context. There are many examples where a rigorous top-down and one-size-fits-all approach did not work. Money was wasted, people became frustrated, and the desired goal was not achieved. It is far better to go slow, pay attention to the local culture, build on what is already there, and use the wisdom of the group. To have a plan $\mathrm{B}$ (and $\mathrm{C}$ and $\mathrm{D}$ ) is always a good idea, while at the same time reminding oneself to be flexible.

\section{Observation 5: implementation of quality improvement is local}

Culture and leadership are local. If culture is defined as "the way things get done around here" the quality improvement measures must be implemented here and now. Quality happens in pockets and then becomes infectious. It is a waste of time and counterproductive to expect instructions from above in order to start thinking about quality or to wait for guidelines from national or international bodies before making the first move.

Local improvement is achievable. Many people have thought about it and put together their ideas and experiences, for example, in the PARIHS framework [6]. The key elements for achieving quality improvements are evidence, context, and facilitation. It is not the quality of the evidence alone that matters, but equally the context where the evidence is to be implemented and how this is done.

The message is: good quality can be achieved. What is needed is an open mind, flexibility, and an awareness that quality implementation depends on the context where it is supposed to happen. Of equal importance is how it is done: paying attention to culture, to communication, and the expectations and previous experiences of those involved. Last but not least, good quality is always cost-effective as it reduces unnecessary expenses and waste, and achieves a good outcome. Keeping in mind the five steps toward better quality will facilitate the implementation of quality measures for the benefit of all involved.

\section{References}

1. Lawson EH, Louie R, Zingmond DS, Brook RH, Hall BL, Han L, Rapp M, Ko CY (2012) A comparison of clinical registry versus administrative claims data for reporting of 30-day surgical complications. Ann Surg 256(6):973-981. doi:10.1097/SLA. 0b013e31826b4c4f

2. Grades of Recommendation, Assessment, Development, and Evaluation (GRADE) Working Group (2004) Grading quality of evidence and strength of recommendations. BMJ 328:1490-1494

3. Kathryn Fitch, Steven J. Bernstein, Mary S. Aguilar, Bernard Burnand, Juan Ramon LaCalle, Pablo Lazaro, Mirjam van het Loo, Joseph McDonnell, Janneke Vader, James P. Kahan (2001) The RAND/UCLA appropriateness method user's manual. RAND, Santa Monica, CA. http://www.rand.org/content/dam/rand/pubs/ monograph_reports/2011/MR1269.pdf. Accessed 13 April 2014

4. Benson K, Hartz AJ (2000) A comparison of observational studies and randomized, controlled trials. N Engl J Med 342(25):1878-1886

5. Concato J, Shah N, Horwitz RI (2000) Randomized, controlled trials, observational studies, and the hierarchy of research designs. N Engl J Med 342:1887-1892

6. Helfrich CD, Damschroder LJ, Hagedorn HJ, Daggett GS, Sahay A, Ritchie M, Damush T, Guihan M, Ullrich PM, Stetler CB (2010) A critical synthesis of literature on the promoting action on research implementation in health services (PARIHS) framework. Implement Sci 5:82. doi:10.1186/1748-5908-5-82 